\title{
Bufotalin sensitizes death receptor-induced apoptosis via Bid- and STAT1-dependent pathways
}

\author{
PORNTHIP WAIWUT, AKIKO INUJIMA, HIROKI INOUE, IKUO SAIKI and HIROAKI SAKURAI \\ Division of Pathogenic Biochemistry, Institute of Natural Medicine, \\ University of Toyama, 2630 Sugitani, Toyama 930-0194, Japan
}

Received June 15, 2011; Accepted August 1, 2011

DOI: $10.3892 /$ ijo.2011.1182

\begin{abstract}
Tumor necrosis factor-alpha (TNF- $\alpha$ ) and TNF-related apoptosis-inducing ligand (TRAIL) are apoptosis-inducing ligands that stimulate death receptors. In this study, we investigated the effects of bufotalin, a major compound in toad venom, on sensitizing TNF- $\alpha$ and TRAIL-induced apoptosis of HeLa cells. Bufotalin promoted death receptor-mediated cell death, especially TRAIL-induced apoptosis, through activation of caspase-3 and PARP-1. Mitochondrial Bid-dependent pathway was activated in TNF- $\alpha$-induced cell death. Cotreatment of bufotalin with TRAIL resulted in the downregulation of antiapoptotic proteins, including Bcl-XL, Mcl-1, survivin and XIAP, and the up-regulation of MAPKs and TRAIL receptor DR5. In addition, phosphorylation of STAT1 was strongly inhibited by bufotalin. Moreover, DR5 expression was induced by knocking down the STAT1 expression. Moreover, the TRAIL-induced apoptotic response was promoted by STAT1 siRNA. Our results demonstrated that bufotalin is a powerful sensitizer of death receptor-induced apoptosis in cancer cells.
\end{abstract}

\section{Introduction}

Many chemotherapeutic agents induce tumor cell death by apoptosis. Apoptosis can be initiated by two distinct pathways: through mitochondria (intrinsic pathway) or through death receptors on the cell surface (extrinsic pathway). Induction of apoptosis via the intrinsic pathway leads to the breakdown of mitochondrial membrane potential and release of cytochrome $\mathrm{c}$,

Correspondence to: Dr Hiroaki Sakurai, Division of Pathogenic Biochemistry, Institute of Natural Medicine, University of Toyama, 2630 Sugitani, Toyama 930-0194, Japan

E-mail: hsakurai@inm.u-toyama.ac.jp

Abbreviations: PARP-1, poly(ADP-ribose) polymerase 1; Bcl-XL, B-cell lymphoma-extra large; Mcl-1, myeloid cell leukemia sequence 1; XIAP, X-linked inhibitor of apoptosis protein; MAPKs, mitogenactivated protein kinases; DR5, death receptor 5; STAT1, signal transducers and activators of transcription-1; siRNA, small interfering RNAs

Key words: TNF- $\alpha$, TRAIL, Bid, STAT1 and subsequently results in the activation of procaspase 9 within the so-called apoptosome (1). In addition, death ligands binding to the death receptors, including Fas, tumor necrosis factor receptor 1 (TNF-R1) or TNF-related apoptosis-inducing ligand (TRAIL) receptor DR4/5, trigger extrinsic apoptosis pathways.

TNF- $\alpha$ binds to TNF-R1, which recruits the adaptor molecule TRADD and FADD to activate a caspase cascade from the upstream initiator caspase- 8 to the downstream effector caspase-3 $(2,3)$. TRAIL is known to induce apoptosis in a variety of tumor cells through its action with two distinct receptors, DR4 and DR5. $\mathrm{Bid}$, a member of the Bcl-2 family, is the main molecular linker connecting the death receptor pathway and the mitochondrial pathway. Bid can activate mitochondria via direct interaction with Bax or Bak. Cytosolic Bid is cleaved by caspase- 8 at the amino terminus to generate a truncated form of Bid (tBid) that mediates cytochrome $\mathrm{c}$ release from mitochondria, which serves as an amplification signal by activating downstream effector caspases, including caspase-3 (4-8). Procaspase-3 can also be directly cleaved by caspase- 8 in a mitochondrion-independent manner. In turn, effector caspases are responsible for cleaving cellular proteins, such as poly(ADP-ribose) polymerase (PARP) (9). Caspase cascade can be blocked by anti-apoptosis proteins, such as cIAP1/2, Bcl-xl, Mcl-1, Survivin and XIAP (10).

Chan $\mathrm{Su}$, toad venom, is a traditional Chinese medicine from the skin secretions of Bufo bufogargarizans Cantor and B. melsanostictus Schneider. It has been used to treat various diseases such as cardiac illness, pain, and cancer in China and other Asian countries $(11,12)$. Bufadienolides, the major active constituents of $\mathrm{Chan} \mathrm{Su}$, are C-24 steroids, the characteristic structural feature of which is a doubly unsaturated six-membered lactone ring ( $\alpha$-pyrone) at position $17 \beta$. Furthermore, these compounds are characterized by the transjunction of rings $\mathrm{B}$ and $\mathrm{C}$ and usually the cis-junction of rings $\mathrm{C}$ and D. More than 40 bufadienolides have been identified, including bufalin, bufotalin, gamabufotalin, cinobufotagin, and resibufogenin $(13,14)$. Bufotalin (Fig. 1A) has been reported to induce apoptosis in human hepatocellular carcinoma (HCC) Hep 3B cells (15); however, its molecular mechanisms in the death receptor signaling pathway remain unknown.

The present study investigated the effect of bufotalin on TNF- $\alpha$ - and TRAIL-induced apoptosis in HeLa cells. We found that activation of the caspase-induced apoptosis pathway by bufotalin was dependent on Bid and STAT1 pathways. 
A

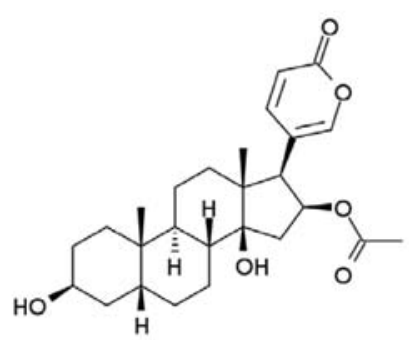

B

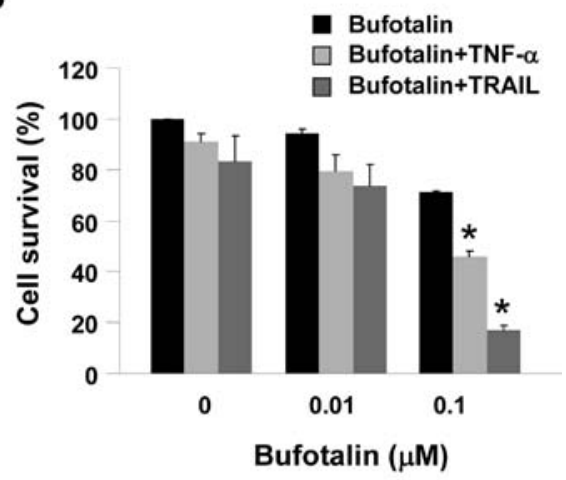

C

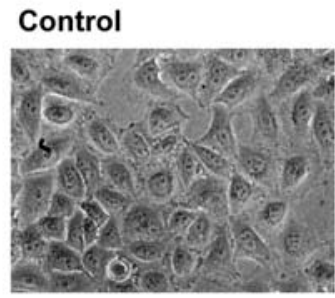

\section{Bufotalin}

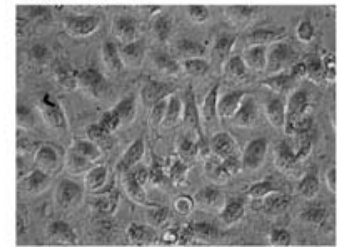

TNF- $\alpha$

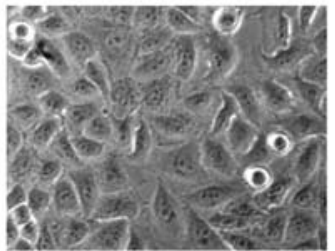

Bufotalin + TNF- $\alpha$

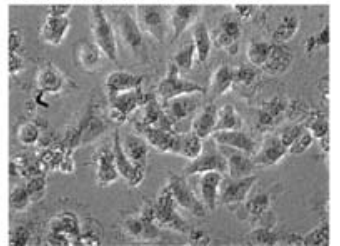

TRAIL

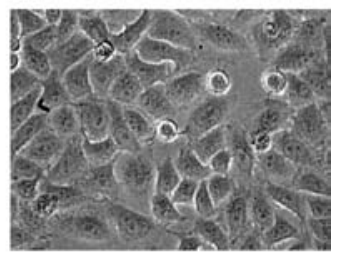

Bufotalin + TRAIL

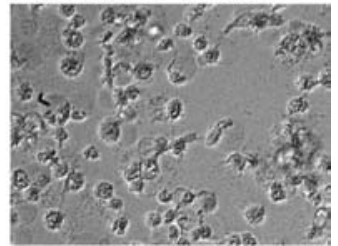

Figure 1. Effects of bufotalin on TRAIL- and TNF- $\alpha$-induced cell death. (A) Chemical structure of bufotalin. (B) HeLa cells were treated with bufotalin at 0.01 and $0.1 \mu \mathrm{M}$ in the presence or absence of TNF- $\alpha(20 \mathrm{ng} / \mathrm{ml})$ or TRAIL $(200 \mathrm{ng} / \mathrm{ml})$ for $24 \mathrm{~h}$. Cell viability was determined by WST-1 assay. ${ }^{*}<<0.001$. (C) Cell morphology was examined in BZ8000 micrographs.

\section{Materials and methods}

Antibodies and reagents. The anti-phospho-specific antibodies against p65 (Ser-536), ERK (Thr-202, Tyr-204), p38 (Thr-180/ Tyr-182), JNK (Thr-183/Tyr-185), STAT1 (Tyr-701), STAT3 (Tyr-705), and total antibodies against PARP-1, Mcl-1, Bid, XIAP Bcl-XL Survivin, STAT3 and caspase-3, -7, -8, -9 were purchased from Cell Signaling Technologies. Antibodies against p38 (C-20-G), JNK, ERK1 (C-16), p65 (C-20-G), STAT1, and actin (C-11) were obtained from Santa Cruz Biotechnology (Santa Cruz, CA, USA). Recombinant human TNF- $\alpha$ was obtained from R\&D Systems. Recombinant human TRAIL was purchased from Peprotech (London, UK). Bufotalin was purchased from Wako Pure Chemical Industries, Ltd. (Osaka, Japan).

Cell culture and cytotoxicity assay. HeLa cells were maintained in Dulbecco's modified Eagle's medium (high glucose) supplemented with $10 \%$ fetal calf serum, $100 \mathrm{U} / \mathrm{ml}$ penicillin and $100 \mu \mathrm{g} / \mathrm{ml}$ streptomycin at $37^{\circ} \mathrm{C}$ in $5 \% \mathrm{CO}_{2}$. Cell viability was quantified using the cell proliferation reagent WST-1 (4-[3-(4-iodophenyl)-2-(4-nitrophenyl)-2H-5-tetrazolio]-1,3-benzene disulfonate) (Dojindo, Kumamoto, Japan). HeLa cells were plated in 96-well microplates at $6 \times 10^{3}$ cells/well, and then incubated for $24 \mathrm{~h}$. Bufotalin-containing medium was added to the wells, and cells were incubated for $30 \mathrm{~min}$, and then stimulated with TNF- $\alpha$. After 24-h incubation, $10 \mu \mathrm{l}$ of WST-1 solution was added, and absorbance was measured at $450 \mathrm{~nm}$.

Small interfering RNAs and transfection. The siRNA for human STAT1 and non-targeting siRNA were purchased from Invitrogen Life Technologies (Carlsbad, CA). HeLa cells were transfected with siRNAs at a final concentration of $20 \mathrm{nM}$ using Lipofectamine reagents (Invitrogen). At $72 \mathrm{~h}$ after transfection, cells were stimulated.

Preparation of cell extracts. Cells were treated with bufotalin and TNF- $\alpha$, and whole cell lysates were prepared with lysis buffer (25 mM HEPES pH 7.7, $0.3 \mathrm{mM} \mathrm{MgCl}_{2}, 0.2 \mathrm{mM}$ EDTA, $10 \%$ Triton $\mathrm{X}-100,20 \mathrm{~mm} \beta$-glycerophosphate, $1 \mathrm{~mm}$ sodium orthovanadate, $1 \mathrm{~mm}$ phenylmethylsulfonyl fluoride (PMSF), $1 \mathrm{~mm}$ dithiothreitol (DTT), $10 \mu \mathrm{g} / \mathrm{ml}$ aprotinin, and $10 \mu \mathrm{g} / \mathrm{ml}$ leupeptin). Cell lysate was collected from the supernatant after centrifugation at $14,000 \mathrm{rpm}$ for $10 \mathrm{~min}$. Nuclear extracts were prepared as described previously (12). In brief, cells were suspended in $420 \mu \mathrm{l}$ buffer A [10 mM HEPES (pH 7.9), $10 \mathrm{mM}$ $\mathrm{KCl}, 0.1$ mM EDTA, 0.1 mM EGTA, 1 mM DTT, 1 mM PMSF, $20 \mathrm{mM} \beta$-glycerophosphate, $0.1 \mathrm{mM}$ sodium orthovanadate, $10 \mu \mathrm{g} / \mathrm{ml}$ aprotinin and $10 \mu \mathrm{g} / \mathrm{ml}$ leupeptin] and chilled on ice for 15 min. Next, $25 \mu 1$ of $10 \%$ Nonidet P-40 was added and 
the suspension was vigorously vortexed for $10 \mathrm{sec}$. The nuclear pellets were suspended in $50 \mu 1$ buffer B (20 mM HEPES (pH 7.9), 0.4 M NaCl, $1 \mathrm{mM}$ EDTA, $1 \mathrm{mM}$ EGTA, $1 \mathrm{mM}$ DTT, $1 \mathrm{mM}$ PMSF, $20 \mathrm{mM} \beta$-glycerophosphate, $1 \mathrm{mM}$ sodium orthovanadate, $10 \mu \mathrm{g} / \mathrm{ml}$ aprotinin and $10 \mu \mathrm{g} / \mathrm{ml}$ leupeptin). The mixture was kept on ice for $15 \mathrm{~min}$ with frequent agitation. Nuclear extracts were prepared by centrifugation at $15,000 \mathrm{rpm}$ for $5 \mathrm{~min}$.

Preparation of mitochondria and cytosol proteins. The cytosolic and mitochondrial protein fractions were isolated according to a previously reported procedure (24). Briefly, after treatment as indicated, cells were scraped in ice-cold homogenization buffer. The cells were then resuspended in 5 volumes of ice-cold extract buffer A (20 mM HEPES, $20 \mathrm{mM} \mathrm{KCl,} 1.5 \mathrm{mM} \mathrm{MgCl} \mathrm{m}_{2}, 1 \mathrm{mM}$ EDTA, 1 mM EGTA, 1 mM DTT, 1 mM PMSF, 1 mM Na $\mathrm{VO}_{3}$, $\mathrm{pH} 7.5$, and $1 \mathrm{X}$ protease inhibitor cocktail) and homogenized. The homogenates were centrifuged at $750 \mathrm{~g}$ for $10 \mathrm{~min}$, and then the supernatant was collected and centrifuged at $10,000 \mathrm{~g}$ for $15 \mathrm{~min}$ to obtain mitochondrion pellets. The supernatants were further centrifuged at $100,000 \mathrm{~g}$ for $1 \mathrm{~h}$ to collect the supernatants (cytosolic fraction).

Immunoblotting. Cell lysate was resolved by SDS-PAGE and transferred to an Immobilon-P-nylon membrane (Millipore). The membrane was treated with BlockAce (Dainippon Pharmaceutical Co. Ltd, Suita, Japan) and probed with primary antibodies. The antibodies were detected using horseradish peroxidase-conjugated anti-rabbit, anti-mouse, and anti-goat IgG (Dako, Glostrup, Denmark), and visualized with the enhanced chemiluminescence system (Amersham Biosciences).

Immunoprecipitation. Cell lysates were diluted with an equal volume of dilution buffer [20 mM HEPES ( $\mathrm{pH} 7.7)$, $2.5 \mathrm{mM} \mathrm{MgCl} 2,0.1 \mathrm{mM}$ EDTA, $0.05 \%$ Triton X-100, $20 \mathrm{mM}$ $\beta$-glycerophosphate, $1 \mathrm{mM}$ sodium orthovanadate, $1 \mathrm{mM}$ phenylmethylsulfonyl fluoride, $1 \mathrm{mM}$ DTT, $10 \mu \mathrm{g} / \mathrm{ml}$ aprotinin, $10 \mu \mathrm{g} / \mathrm{ml}$ leupeptin]. After centrifugation, lysates were incubated with anti-DR4 and DR5 antibody (Santa Cruz Biotechnology) on ice for $1.5 \mathrm{~h}$ and then rotated with protein $\mathrm{G}-$ Sepharose (Amersham Biosciences) at $4^{\circ} \mathrm{C}$ for $2 \mathrm{~h}$. The Sepharose beads were washed twice with washing buffer (a 1:1 mixture of whole-cell lysate buffer and dilution buffer).

Real-time RT-PCR. Total RNA was prepared using the RNeasy Mini kit (Qiagen). First-strand cDNA was synthesized by SuperScript II reverse transcriptase (Invitrogen). The cDNA was amplified quantitatively using SYBR Premix Ex Taq (Takara-Bio, Otsu, Japan), using the following primers: DR5 sense: 5'-GCACCACGACCAGAAA-3'; antisense: 5'-CACC GACCTTGACCAT-3'. Real-time quantitative RT-PCR was performed using an ABI PRISM 7300 sequence detection system (Applied Biosystems, Foster City, CA). All data were normalized to GAPDH mRNA.

\section{Results}

Bufotalin sensitizes HeLa cells to TRAIL and TNF- $\alpha$-induced cell death. We first examined the effects of bufotalin on TNF- $\alpha$ and TRAIL-induced cytotoxicity (Fig. 1B). HeLa cells were resistant

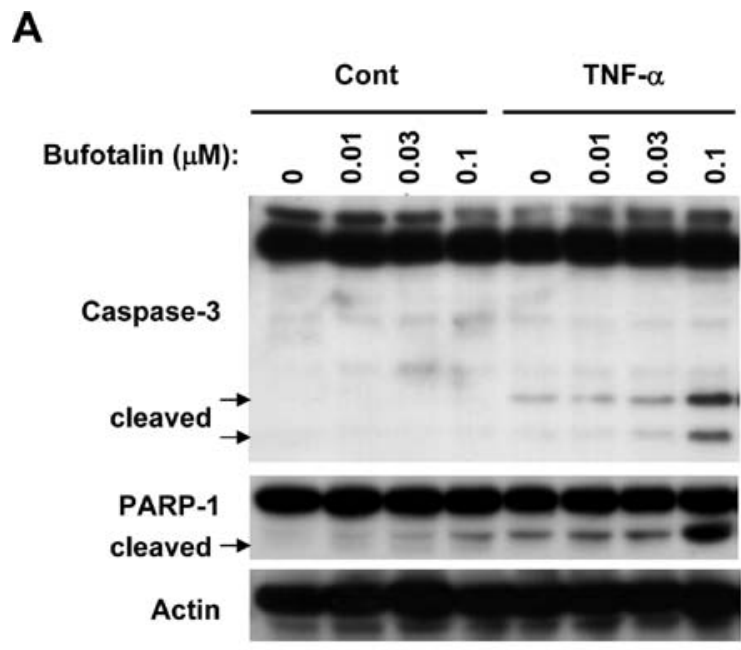

B

C
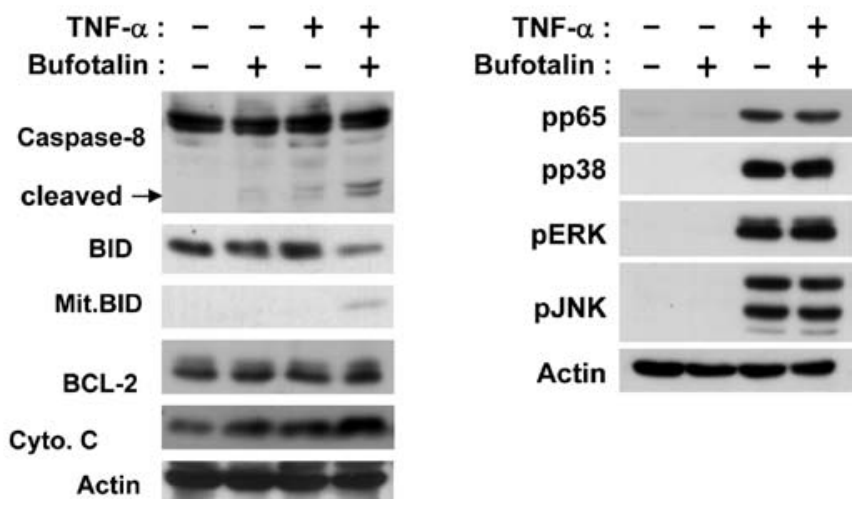

Figure 2. Effects of bufotalin on TNF- $\alpha$-induced apoptosis. HeLa cells were pretreated with bufotalin at various concentrations $(0.01,0.03$ and $0.1 \mu \mathrm{M})$ for $30 \mathrm{~min}$, and then stimulated with TNF- $\alpha(20 \mathrm{ng} / \mathrm{ml})$ for $4 \mathrm{~h}$. Whole cell extract was prepared, fractionated and analyzed by Western blotting. (A) Western blot results using anti-caspase-3, PARP and actin antibodies. Arrows indicate cleaved forms of caspases-3 and PARP. (B) Effects of bufotalin on TNF$\alpha$-mediated Bid-dependent pathway. Cells were pretreated with bufotalin at $0.1 \mu \mathrm{M}$ for $30 \mathrm{~min}$, and then stimulated with TRAIL $(200 \mathrm{ng} / \mathrm{ml})$ for $4 \mathrm{~h}$. Western blotting performed by using anti-caspase-8, Bid, BCL-2, cytochrome c, and actin antibodies. (C) Effects of bufotalin on TNF- $\alpha$-induced NF- $\kappa$ B and MAPK signaling pathways were examined using anti-phospho-p65, Erk, p38, JNK and actin antibodies.

to TNF- $\alpha$ - and TRAIL-induced cell death. Bufotalin at $0.1 \mu \mathrm{M}$ alone showed weak cytotoxicity in a WST-1 assay; however, combination with TNF- $\alpha$ or TRAIL markedly increased the sensitivity to these cytokines. Phase-contrast microscopy supported the results of a proliferation assay (Fig. 1C). The results indicate that bufotalin markedly enhanced both TNF- $\alpha$ and TRAIL-induced cancer cell deaths.

Bufotalin enhances TNF- $\alpha$-induced apoptosis through Bid-dependent pathway. We investigated the effect of bufotalin on TNF- $\alpha$-induced apoptosis by determining the cleavage of caspase-3 and PARP-1. Cells were pretreated with bufotalin at various concentrations for $30 \mathrm{~min}$, and then stimulated with TNF- $\alpha$ for $4 \mathrm{~h}$. TNF- $\alpha$ alone slightly induced the cleavage of caspase- 3 and PARP-1; however, bufotalin enhanced TNF- $\alpha$ induced cleavage in a concentration-dependent manner (Fig. 2A). The combined treatment also induced cleavage of caspase- 8 


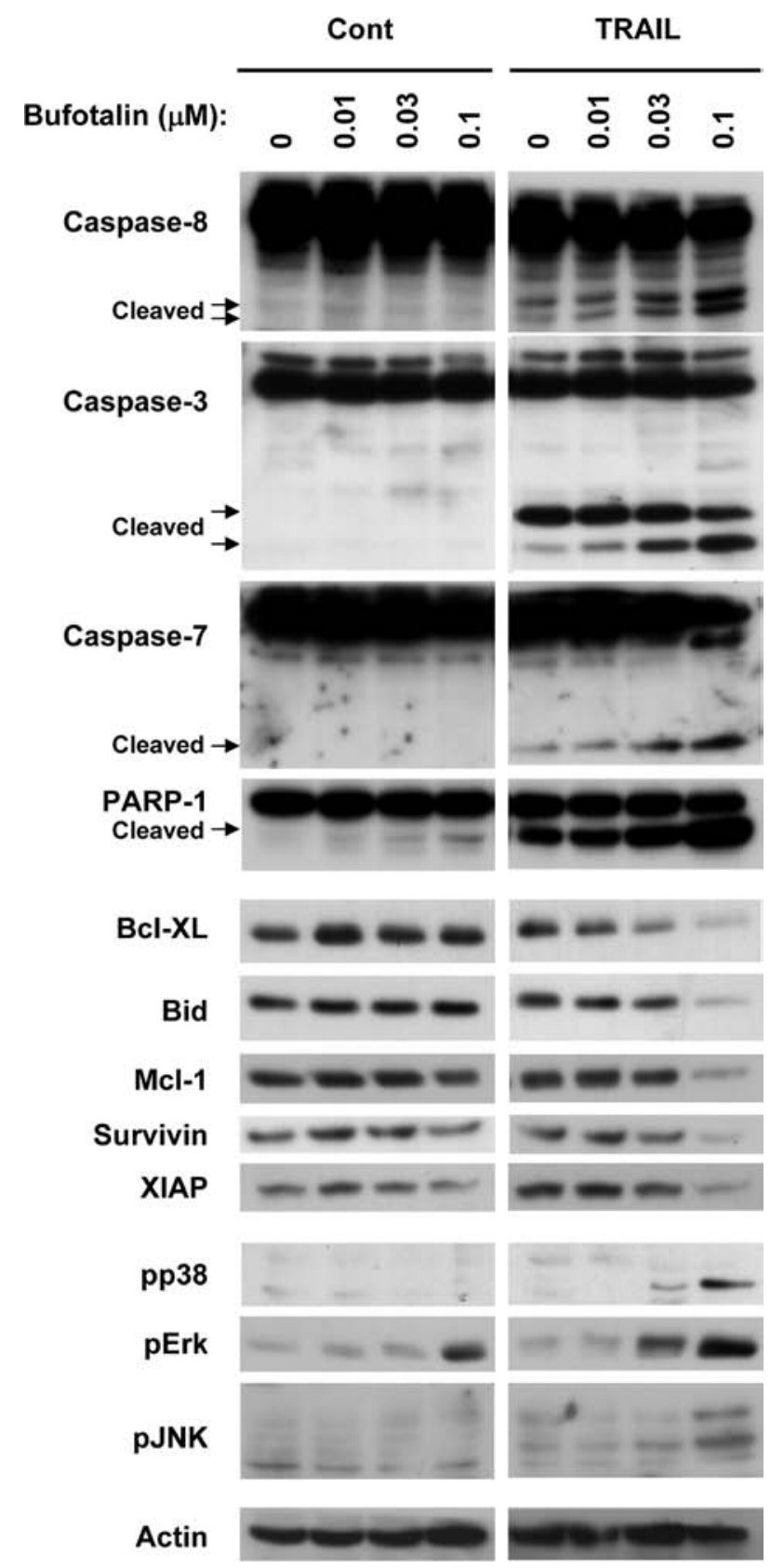

Figure 3. Effects of bufotalin on TRAIL-induced apoptosis. HeLa cells were pretreated with bufotalin at indicated concentrations for $30 \mathrm{~min}$, and then stimulated with TRAIL $(200 \mathrm{ng} / \mathrm{ml})$ for $4 \mathrm{~h}$. Whole cell extract was prepared, fractionated and analyzed by Western blotting using anti-caspase- $8,-3,-7$, PARP, Bcl-XL, Bid, Mcl-1, Survivin, XIAP, anti-phospho Erk, p38, JNK and anti-actin antibodies. Arrows indicate cleaved forms of caspases and PARP.

(Fig. 2B). In addition, mitochondrial-type Bid and subsequent cytochrome c release were increased. In contrast, activation of NF- $\mathrm{kB}$ p65 and three MAPK cascades (p38, Erk and JNK) was not affected (Fig. 2C). This result suggested that bufotalin potentiated TNF- $\alpha$-induced apoptosis through a Bid-dependent pathway.

Bufotalin sensitizes TRAIL-induced apoptosis. Similar to the effect on TNF- $\alpha$ response, bufotalin promoted TRAIL-induced cleavage of caspase- $8,-3,-7$ and PARP-1 in a concentrationdependent manner (Fig. 3). In addition, the expressions of
A
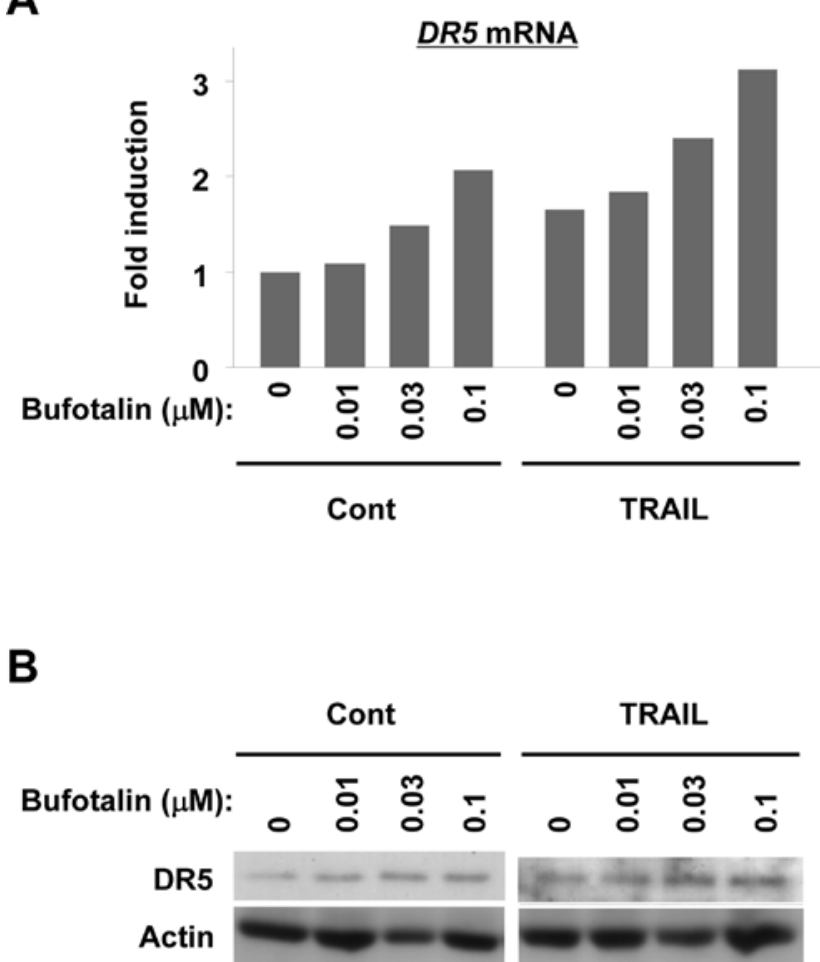

Figure 4. Effects of bufotalin on TRAIL-induced DR5 expression. HeLa cells were pretreated with bufotalin at the indicated concentrations for $30 \mathrm{~min}$, and then stimulated with TRAIL (200 ng/ml) for $4 \mathrm{~h}$. (A) Real-time PCR analysis to ascertain the expression of DR5 mRNA. (B) Whole cell extract was prepared, fractionated and analyzed by Western blotting using anti-DR5 and actin antibodies.

anti-apoptotic proteins Bcl-XL, Mcl-1, Survivin and XIAP were reduced markedly by treatment with both TRAIL and bufotalin. Moreover, total Bid, a mitochondrion-dependent apoptotic protein, was also decreased. Because MAPKs and NF- $\kappa B$ play important roles in modulating apoptosis, we determined if such signaling pathways are involved in bufotalin-induced cell death. Consequently, although treatment with TRAIL or bufotalin alone had no effect on the phosphorylated forms of p38, ERK, and JNK, the combination of TRAIL and bufotalin resulted in synergistic activation of p38, ERK, and JNK at $4 \mathrm{~h}$ (Fig. 3). These results indicated that bufotalin synergized the decrease in anti-apoptotic protein expression in the presence of TRAIL.

Effect of bufotalin on the expression of TRAIL receptor DR5. In order to ascertain the mechanism by which bufotalin sensitizes HeLa cells to TRAIL, we next investigated whether the compound enhances the expression of death receptors; therefore, cells treated with bufotalin and TRAIL were analyzed by RT-PCR to quantify DR5 mRNA expresison. As shown in Fig. 4A, 4-h incubation with bufotalin alone slightly enhanced DR5 mRNA expression in a dose-dependent manner and TRAIL enhanced this activity. Fig. 4B demonstrated that DR5 protein expression was also up-regulated by bufotalin and TRAIL.

Bufotalin inhibits TRAIL-induced STAT1 phosphorylation. It has been reported that JAK and STAT pathways could suppress apoptosis by inhibiting DR 5 expression. In the present study we 
A

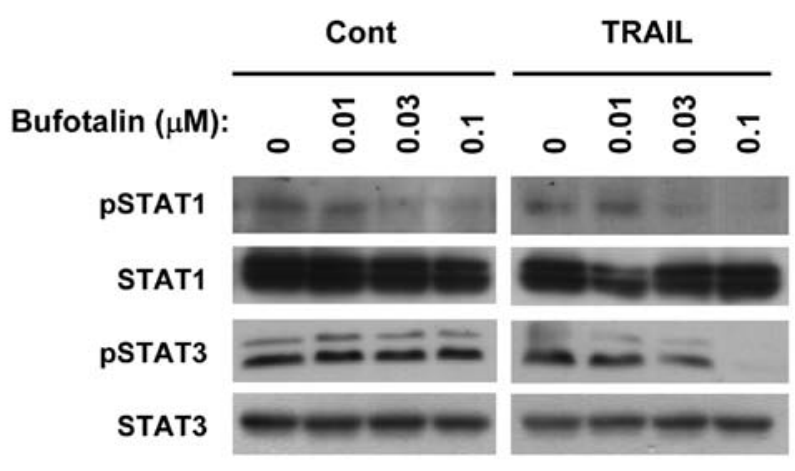

B

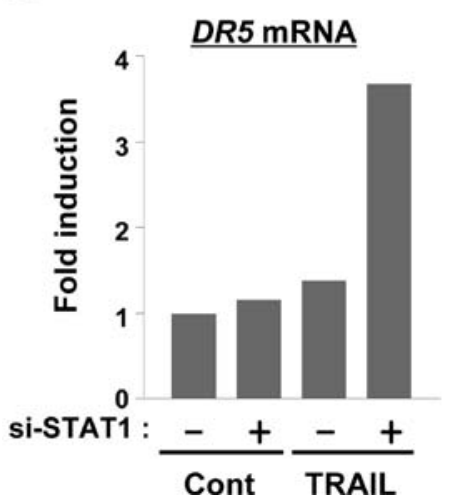

C

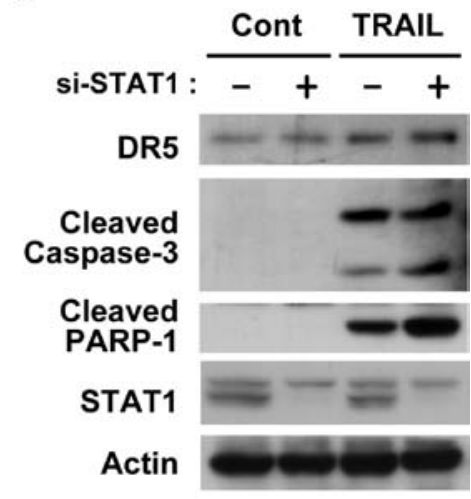

Figure 5. Effects of bufotalin on TRAIL-induced STAT1 pathway. (A) HeLa cells were pretreated with bufotalin at the indicated concentrations for 30 min, and then stimulated with TRAIL (200 ng/ml) for $4 \mathrm{~h}$. Whole cell extract was prepared, fractionated and analyzed by Western blotting using anti-phospho STAT1, STAT-3 and anti- STAT1, STAT3 antibodies. (B) HeLa cells were transfected with Luc and STAT1 siRNA and then treated or untreated with TRAIL (200 ng/ml) for $4 \mathrm{~h}$. (B) Real-time PCR analysis to ascertain the expression of DR5 mRNA. (C) Whole cell extract was prepared, fractionated and analyzed by Western blotting using anti-STAT1, DR5, caspase-3, PARP-1 and actin antibodies.

investigated the effects of bufotalin on TRAIL-mediated phosphorylation of STAT1 and STAT3. Fig. 5A shows that cotreatment with bufotalin and TRAIL suppressed the phosphorylation of both STAT1 and STAT3 in a concentration-dependent manner. Since TRAIL signaling is often accompanied by the expression of DR5, we next investigated the functional significance of STAT1 in sensitization to TRAIL. DR5 mRNA expression was strongly induced by TRAIL in cell down-regulating STAT1 expression (Fig. 5B). In addition, TRAIL-induced caspase-3 and PARP activation was enhanced by STAT1 knock-down, indicating that down-regulation of STAT1 is sufficient to sensitize cells to TRAIL-induced apoptosis.

\section{Discussion}

Bufotalin, one of the bufadienolides isolated from Formosan $\mathrm{Chan} \mathrm{Su}$, has been used as an important traditional Chinese medicine for heart failure and pain. Bufadienolides are the major bioactive constituents of Chan $\mathrm{Su}$, exhibited strong anticancer, cardiotonic and anesthetic activities (12). In particular, they have significant cytotoxic activities against various human cancer cells, such as gastric and liver cancer cells $(16,17)$. A previous study reported that bufotalin induced apoptosis of Hep 3B cells (15); however, the mechanism of bufotalin for death-receptorinduced apoptosis is not clearly understood. Consequently, this study investigated the effects and molecular mechanisms of bufotalin in sensitizing TNF- $\alpha$ and TRAIL-mediated apoptosis of HeLa cells.

Death receptor-mediated tumor cell death, either alone or in combination with other anticancer drugs, is considered a new approach for anticancer therapy. TNF- $\alpha$ and TRAIL are death ligands playing an important role in apoptosis induction through their death receptor pathways (3). In the present study, we found that bufotalin markedly enhanced TRAIL and TNF- $\alpha$-induced cell death through caspase-3 and PARP-1 activation.

In the death receptor-mediated apoptosis signaling pathway, $\mathrm{NF}-\kappa \mathrm{B}$ is widely known for its ubiquitous roles in cell survival and apoptosis. NF- $\kappa \mathrm{B}$ protects cells from apoptosis by transcriptional activation of survival factors, such as anti-apoptotic proteins (18). Our results showed that bufotalin had no effect on TNF- $\alpha$ - and TRAIL-induced NF- $\kappa$ B activation (data not shown). In other pathways, the contribution of MAPKs has been extensively documented. As indicated previously, p38 ERK and JNK activation leads to the mitochondrial apoptotic pathway $(19,20)$, and JNK activation promotes the processing of Bid to a proapoptotic cleaved product (21). Previous experiments have reported that JNK ERK and p38 activation contributed to the sensitization of TRAIL-induced apoptosis (22-24). Our results indicated that co-treatment with bufotalin and TRAIL resulted in enhanced apoptosis through delayed activation of 
MAPKs, whereas there was no effect of bufotalin on TNF- $\alpha$ induced rapid activation of MAPKs. As bufotalin has no effect on TNF- $\alpha$-induced NF- $\kappa \mathrm{B}$ and MAPKs, we therefore suggest that the signaling pathway may be involved in the mitochondrial apoptotic pathway. In our study, bufotalin induced TNF-induced cleavage of caspase- 3 and PARP-1 through Bid activation, indicating the participation of mitochondrion dependent apoptotic machinery in cell death.

Previous it was reported that a decrease in the phosphorylated form of STAT3 could be responsible for the enhanced expression of death receptor (25). In this study, we found that bufotalin together with TRAIL inhibited STAT1 and STAT3 phosphorylation, and induced DR5 mRNA and protein expression, indicating that bufotalin may enhance TRAIL-induced death receptor expression through STAT3. Moreover, we found that downregulation of STAT1 with siRNA caused upregulation of DR5 mRNA and the protein level, which was sufficient to sensitize cells to TRAIL-induced apoptosis. This finding indicated that bufotalin sensitizes TRAIL-induced apoptosis via STAT1-mediated DR5 upregulation. To further study the mechanism of bufotalin-induced apoptosis, both anti-apoptotic molecules (Bcl-XL, Mcl-1, Survivin and XIAP) and the proapoptotic molecules Bid were examined in this study. Among them, we demonstrated that co-treatment with bufotalin and TRAIL was able to downregulate Bcl-XL, MclL-1, Survivin XIAP and Bid.

In conclusion, bufotalin can sensitize both TNF- $\alpha$ - and TRAIL-induced apoptosis in HeLa cells. Bufotalin participated in TNF- $\alpha$-induced apoptosis via a Bid-dependent pathway by activating caspase- 8 , Bid, cytochrome $\mathrm{c}$ and caspase- 3 , respectively, whereas bufotalin sensitized TRAIL-induced apoptosis through MAPK activation and STAT1-mediated upregulation of DR5.

\section{Acknowledgements}

This work was supported in part by Grants-in-Aid for Challenging Exploratory Research (no. 09002374) from the Ministry of Education, Culture, Sports, Science and Technology (MEXT), Japan, and a grant from the First Bank of Toyama Foundation.

\section{References}

1. Fulda S and Debatin KM: Extrinsic versus intrinsic apoptosis pathways in anticancer chemotherapy. Oncogene 25: 4798-4811, 2006.

2. Ding WX and Yin XM: Dissection of the multiple mechanisms of TNF-alpha induced apoptosis in liver injury. J Cell Mol Med 8: 445-454, 2004.

3. Smith CA, Farrah T and Goodwin RG: The TNF receptor superfamily of cellular and viral proteins: activation, costimulation, and death. Cell 76: 959-962, 1994.

4. Kantari $\mathrm{C}$ and Walczak H: Caspase- 8 and Bid: caught in the act between death receptors and mitochondria. Biochim Biophys Acta 1813: 558-563, 2011

5. Chen X, Ding WX, Ni HM, Gao W, Shi YH, Gambotto AA, Fan J, Beg AA and Yin XM: Bid-independent mitochondrial activation in tumor necrosis factor alpha-induced apoptosis and liver injury. Mol Cell Biol 27: 541-553, 2006.
6. Perez D and White E: TNF-alpha signals apoptosis through a bid-dependent conformational change in Bax that is inhibited by E1B 19K. Mol Cell 6: 53-63, 2000.

7. Stevenson HS, Fu SW, Pinzone JJ, Rheey J, Simmens SJ and Berg PE: BP1 transcriptionally activates bcl-2 and inhibits TNFalpha-induced cell death in MCF7 breast cancer cells. Breast Cancer Res 9: R60, 2007.

8. Pei Y, Xing D, Gao X, Liu L and Chen T: Real-time monitoring full length bid interacting with Bax during TNF-alpha-induced apoptosis. Apoptosis 12: 1681-1690, 2007.

9. Alvarez S, Blanco A, Fresno M and Muñoz-Fernández MÁ: TNF- $\alpha$ contributes to caspase- 3 independent apoptosis in neuroblastoma cells: role of NFAT. PLoS One 6: e16100, 2011.

10. Thorburn A, Behbakht K and Ford H: TRAIL receptor-targeted therapeu-tics: resistance mechanisms and strategies to avoid them. Drug Resist Updat 11: 17-24, 2008.

11. Xu W, Luo H, Zhang Y, Shan L, Li H, Yang M, Liu R and Zhang W: Simultaneous determination of five main active bufadienolides of Chan $\mathrm{Su}$ in rat plasma by liquid chromatography tandem mass spectrometry. J Chromatogr B Analyt Technol Biomed Life Sci 859: 157-163, 2007.

12. Puschett JB, Agunanne E and Uddin MN: Emerging role of the bufadienolides in cardiovascular and kidney diseases. Am J Kidney Dis 56: 359-370, 2010.

13. Li J, Ma X, Li F, Wang J, Chen H, Wang G, Lv X, Sun C and Jia J: Preparative separation and purification of bufadienolides from Chinese traditional medicine of ChanSu using high-speed counter-current chromatography. J Sep Sci 33: 1325-1330, 2010.

14. Nogawa T, Kamano Y, Yamashita A and Pettit GR: Isolation and structure of five new cancer cell growth inhibitory bufadienolides from the Chinese traditional drug Ch'an Su. J Nat Prod 64: 1148-1152, 2001.

15. Ye, M.and Guo DA: Analysis of bufadienolides in the Chinese drug ChanSu by high-performance liquid chromatography with atmospheric pressure chemical ionization tandem mass spectrom-etry. Rapid Commun Mass Spectrom 19: 1881-1892. 2005.

16. Su CL, Lin TY, Lin CN and Won SJ: Involvement of caspases and apoptosis-inducing factor in bufotalin-induced apoptosis of Hep 3B cells. J Agric Food Chem 57: 55-61, 2009.

17. Watabe M, MasudaY, Nakajo S, Yoshida T, Kuroiwa Y and Nakaya K: The cooperative interaction of two different signaling pathways in response to bufalin induces apoptosis in human leukemia U937 cells. J Biol Chem 271: 14067-14072, 1996.

18. Luo J, Kamata $\mathrm{H}$ and Karin M: IKK/NF- $\kappa B$ signaling: balancing life and death- a new approach to cancer therapy. J Clin Invest 115: 2625-2632, 2005.

19. Garcia-Fernandez LF, Losada A and Alcaide V: Aplidin induces the mitochondrial apoptotic pathway via oxidative stress-mediated JNK and p38 activation and protein kinase Cy. Oncogene 21: 7533-7544, 2002.

20. Capano M and Crompton M: Bax translocates to mitochondria of heart cells during simulated ischaemia: involvement of AMP-activated and p38 mitogen-activated protein kinases. Biochem J 395: 57-64, 2006.

21. Deng Y, Ren X, Yang L, Lin Y and Wu X: A JNK-dependent pathway is required for TNF-induced apoptosis. Cell 115: 61-70, 2003.

22. Lin FL, Hsu JL, Chou CH, Wu WJ, Chang CI and Liu HJ: Activation of p38 MAPK by damnacanthal mediates apoptosis in SKHep 1 cells through the DR5/TRAIL and TNFR1/TNF- $\alpha$ and p53 pathways. Eur J Pharmacol 650: 120-129, 2011.

23. Nordström E, Fisone $\mathrm{G}$ and Kristensson K: Opposing effects of ERK and p38-JNK MAP kinase pathways on formation of prions in GT1-1 cells. FASEB J 23: 613-622, 2008.

24. Nesterov A, Nikrad M, Johnson T and Kraft AS: Oncogenic ras sensitizes normal human cells to tumor necrosis factor- $\alpha$-related apoptosis-inducing ligand-induced apoptosis. Cancer Res 64: 3922-3927, 2004

25. Carlisi D, D'Anneo A, Angileri L, Lauricella M, Emanuele S, Santulli A, Vento R and Tesoriere G: Parthenolide sensitizes hepatocellular carcinoma cells to TRAIL by inducing the expression of death receptors through inhibition of STAT3 activation. J Cell Physiol 226: 1632-1641, 2010. 